\title{
Interactive comment on "Effect of neutral winds on the creation of non-specular meteor trail echoes” by Freddy Galindo et al.
}

\section{Freddy Galindo et al.}

jvu1@psu.edu

Received and published: 13 January 2021

Authors' response to the comments of reviewer 2:

We thank the reviewer for the suggestions and comments. Below, we present our responses to each of those comments. We have repeated the relevant comments for convenience in each response and then provide the text (in blue) we intend to add in the revised manuscript.

General Comments: This manuscript presents an interesting analysis of radar observations of meteors and the effects of winds on these. It is trying to answer an important question in meteor physics: what are the effects of winds on wave growth in meteor trails and, hence, on non-specular radar detections. It uses a simple model devel- 
oped more than 15 years ago and applies it to a range of atmospheric characteristics. However, as explained in the comments made below this manuscript leaves a lot of unanswered questions.

Response: Below are the points we want to address in this study. We will add these points to the revised version of the manuscript.

Interactive

comment

- Our model and the associated software can be executed in a general-purpose PC-based system. It can easily be adapted and combined with other tools to study very large meteor populations. In contrast, as far as we know, more sophisticated 3D meteor models require supercomputer clusters and do not fully simulate the actual extent of a meteor trail or produce results that can be closely compared to $2 \mathrm{D}$ observational data.

- Although our numerical model is a simplified representation of the meteor physics, it can produce very good and fine details such as those reported in this paper. Our model can be used to account for and understand the statistical outcome of thousands of meteors acting collectively on the Earth's Upper Atmosphere.

- We plan to fine-tune our meteor model and plan to make the code open-source to the scientific community so others can verify our findings or expand our results. We envision our efforts not to replace but complement more complex 3D meteor models.

Major Comments: 1) Extensive observations at JRO (See Sugar et al, 2010 and Oppenheim et al, 2009) seem to show that, as trails span many km (sometimes over $15 \mathrm{~km}$ ), through regions of the atmosphere with vastly different wind speeds trails seem to develop regardless of wind speed. This is odd because simulations and theory, like that presented in this manuscript and more recent ones, imply one would expect that wave growth would depend on wind speed. This may result from drifts and currents 
that travel along the length of the trail, making the local wind speed less important to wave growth.

ANGEOD

Response: This is a very important observation, and we partially agree with the reviewer. We are developing a deep learning algorithm to detect and classify thousands of meteors properly. Once this algorithm is fully functional, we plan to expand the results reported in this manuscript and perform an extensive statistical analysis of trail echoes with gaps.

2) Line 50: The background has no references past 2011 but there has been extensive progress made on topics immediately relevant to the manuscript since then.

Response: We will add these additional references:

1. Oppenheim, M. M., S. Arredondo, and G. Sugar (2014), Intense winds and shears in the equatorial lower thermosphere measured by high-resolution nonspecular meteor radar, J. Geophys. Res. Space Physics, 119, 2178-2186, doi:10.1002/2013JA019272.

2. Oppenheim, M. M., and Y. S. Dimant(2015), First 3-D simulations of meteor plasma dynamics and turbulence, Geophys. Res. Lett., 42, 681-687, doi:10.1002/2014GL062411

3. Dimant, Y. S. and M. M. Oppenheim(2017), Formation of plasma arounda small meteoroid: 1. Kinetic theory,J. Geophys. Res. Space Physics,122, 46694696,doi:10.1002/2017JA023960.

4. Chau, J. L., Strelnikova, I., Schult, C., Oppenheim, M. M., Kelley, M. C., Stober, G., and Singer, W. (2014), Nonspecular meteor trails from nonâǍŔfieldâĂŘaligned irregularities: Can they be explained by presence of charged meteor dust?, Geophys. Res. Lett., 41, 3336- 3343, doi:10.1002/2014GL059922..

5. Dimant, Y. S., and Oppenheim, M. M. (2017), Formation of plasma around a small meteoroid: 2. Implications for radar head echo, J. Geophys. Res. Space Physics, 122, 4697-4711, doi:10.1002/2017JA023963.

Interactive

comment 
6. Sugar, G., Oppenheim, M. M., Dimant, Y. S., Close, S. (2018). Formation of plasma around a small meteoroid: Simulation and theory. Journal of Geophysical Research: Space Physics, 123, 4080- 4093. https://doi.org/10.1002/2018JA025265

7. Sugar, G., Oppenheim, M. M., Dimant, Y. S., Close, S. (2019). Formation of plasma around a small meteoroid: Electrostatic simulations. Journal of Geophysical Research: Space Physics, 124, 3810-3826. https://doi.org/10.1029/2018JA026434

3) Line 55 Correct me if I'm wrong but this model is not exactly the state of the art and is a highly simplified 2-D model of a 3-D phenomenon. It may catch the basic physics but still, the authors should look into the more sophisticated models of instability for a 3-D meteor plasma of Dimant, et al (2015-2017). Also, the 3-D simulations of Oppenheim et. Al (2015).

Response: Rather than attempting to reproduce these more complex 3D simulations pointed out by the reviewer, our research efforts are complementary to this comment. In the response to the general comments, we provided the main points we seek to address with this study. We will include these relevant references pointed out by the reviewer in the revised version of the manuscript.

4) Line 167: These observations are interesting and help to make this case. However, when we've examined high-resolution images containing both head and trail echoes, we've generally seen that the heads and trails have gaps at the same ranges, implying that reduced returns were due to the nulls. Also, there are 2 papers where wind data was inferred from trail echoes and those winds go through zero and they still returned trail echoes. I agree that I would expect weaker trails when the winds are small but that is not what we have seen observationally. The field needs a larger statistical analysis of data comparing head and trail gaps to really see what the pattern is. A handful of cases will not be compelling either way because of the complexity and noise in this data.

Response: We agree with the reviewer that antenna nulls and noise levels in the data 
could be reasonable explanations to account for certain meteor events when gaps are observed in both the head and trail echoes at the same ranges. We are developing a deep learning algorithm to detect and classify automatically thousands of meteors properly near real-time. Once this algorithm is fully functional, we plan to expand the results reported in this manuscript and perform an extensive statistical analysis of trail echoes with gaps. We also expect to carry out future radar experiments and compute neutral wind amplitudes using meteor trails as described in (Oppenheim et al., 2009) to establish a complete understanding of the gaps shown in this paper. We will add this text near line 167 to address some of these comments.

Routine meteor interferometry analysis, described in (Chau and Galindo, 2008), was applied to the events presented in this paper. We analyzed each of the head echo events using both received SNR and interferometry analysis. We discarded noise level as a potential explanation since in the examples we report, all trail echoes were at least $3 \mathrm{~dB}$ above the noise level. So statistically, it is improbable that noise is responsible for gaps in different echoes at the same range and around the same time. We also discarded antenna nulls as a possible explanation for the trails' gaps since interferometry analysis placed these events in the main lobe of the antenna. These examples from Jicamarca have echo gaps observed only in the trail echoes, as shown in Figure 8a. Notice that there is no drop in power intensity for the head echo around the $104.5 \mathrm{~km}$ range when zoom-in in this figure.

5) Line 168: This image seems to show a gap in both head and trail, though the head isn't well resolved. Fig. 8a though does seem to show a reasonably strong head but a gap in the trail. This is intriguing but a single case is not sufficient. The Oppenheim, 2013 JGR shows that we do often get echoes at low velocities.

Response: Figure 7 shows two examples collected with a medium power VHF radar (Urbina et al., 2000), while Figure 8 displays two events probed with Jicamarca HPLA radar. Like we indicate in the manuscript near line 180 that we found 17 (out of 103). These events were manually classified and analyzed. Since this approach is quite

Interactive comment 
tedious, we are currently developing a deep learning algorithm to detect and classify thousands of meteors correctly in almost real-time. Once this algorithm is fully functional, we plan to expand the results reported in this manuscript and perform an extensive statistical analysis of trail echoes with gaps.

6) Line 219: The gap between the trail and the head is usually fairly constant or changing slowly, more so than the winds typically do.

Interactive

comment

Response: We agree with the reviewer. This is why we showed this simulation to illustrate how low values neutral winds can produce these head-trails pairs with larger gaps. In practice, detecting a trail echo also depends on other factors such as the radar transfer function, receiver bandwidth, noise, etc.

\section{Minor Comments:}

Line 19: "Past decade" -> at least two decades now (Chapin and Kudeki is over 25 years...)

Response: We have changed this expression to For more than two decades.

Line 65: A summary of what physics is and is not in this model would be helpful to readers so they need not return to these 3 papers. I believe you could say it's a 2-D local theory that assumes an infinite homogeneous trail and background (or something similar). It neglects physics along $B$ or inhomogeneities of any kind. Or something similar.

Response: We will modify the text between lines 55-60 to provide a summary of meteor physics included in the model as follows.

As explained in these papers, the model starts by computing the amount of ablated particles created behind the meteoroid body. These energetic particles are then used to calculate the amount of ionization made in the trail. Here we assume that the ionization created in the trail is initially distributed in a cylindrical volume defined by the initial radius. At this point, the trail is expanded by either ambipolar diffusion or tur西 
bulent diffusion to simulate the absence or presence of plasma instabilities in the trail during its evolution (Dyrud et al., 2001; Yee and Close, 2013). The plasma instability analysis is based on meteor Farley-Buneman Gradient-Drift (FBGD) instability reported in Dyrud et al. (2002), Oppenheim et al. (2001), and Oppenheim et al. (2003). NSMES assumes that a non-specular meteor trail echo is created because the trail becomes Bragg reflective at altitudes, where plasma instabilities can develop (Dyrud et al., 2002). The simulations produce artificial radar range-time-intensity (RTI) images that we use as proxies to help us in the analysis of Coqui-II and Jicamarca meteor observations.

Line 167: The word "considerable" is too vague.

Response: We have replaced this term with more than 1000

Line 184: This implies this was a skimmer. Is that right?

Response: Both meteor events shown in Figure 8 exhibit elevation angles around 70 degrees and appear to traverse the main beam.

Line 186: "below or above" means all of them? And the feature referred to is unclear.

Response: Yes, we meant the remaining meteors observed around this period of time do not exhibit the feature under discussion. We will remove the sentence since the authors believe it isn't necessary and is confusing the reader.

We will delete: "Meteor trails observed below or above $104.5 \mathrm{~km}$ altitude do not exhibit this feature."

Line 192: 11 years is not so recent.

Response: We will remove the term "recent paper."

Printer-friendly version

Line 204: These LATE flares also seem to effect the head echoes, are not all at low altitudes and are quite rare (while wind shears are not). 
Response: We agree that wind shears aren't rare. However, the proper combination of physical parameters to create a FLARE event based on our findings represent a rare situation. We will clarify these comments in the paper by editing lines $204-207$ as follows:

Notice that the creation of LATE events based on our findings requires an unusual combination of meteoroid occurrence and atmosphere background conditions, making LATE events rare. However, our results are not only limited to the final stages of the meteoroid occurrence. We also expect to see LATE-like events at the initial stage of the meteoroid passage if the right conditions, such as background electron density, winds, etc., are satisfied.

Line 214: Divergence not "divergent"

Response: We agree, it should be "divergence."

Interactive comment on Ann. Geophys. Discuss., https://doi.org/10.5194/angeo-2020-41, 2020. 